\title{
The Generation of Quantum Objects by Quantum Loops
}

\author{
Hans H. Diel \\ Diel Software Beratung und Entwicklung, Sindelfingen, Germany \\ Email: www.hans-diel.de/physics.htm, diel@netic.de
}

How to cite this paper: Diel, H.H. (2019) The Generation of Quantum Objects by Quantum Loops. Open Access Library Journal, 6: e5700.

https://doi.org/10.4236/oalib.1105700

Received: August 12, 2019

Accepted: September 21, 2019

Published: September 24, 2019

Copyright ( 2019 by author(s) and Open Access Library Inc.

This work is licensed under the Creative Commons Attribution International License (CC BY 4.0).

http://creativecommons.org/licenses/by/4.0/

(c) (i) Open Access

\begin{abstract}
Ultimate goal of the work described in this paper has been the construction of a local causal model of quantum theory including quantum field theory. The original model has been refined in a number of refinement steps. As the so far latest refinement step, quantum loops are identified as the elementary processes of spacetime dynamics and quantum field dynamics. Quantum loops are processes that constitute quantum objects. In the causal model of quantum loops and quantum objects presented here, the collective behavioral processes involve the elementary units of spacetime and the associated elementary units of quantum of fields. As such, quantum loop processes are the sources of gravitational fields (i.e., spacetime curvature) and of the quantum objects (wave function). The model may be viewed as a derivative of loop quantum gravity, spin networks and causal dynamical triangulation, although significant deviations to these theories exist. The causal model of quantum loops is based on a causal model of spacetime dynamics where space (-time) consists of interconnected space points, each of which is connected to a small number of neighboring space points. The curvature of spacetime is expressed by the density of these space points and by the arrangement of the connections between them. The quantum loop emerges in a collective behavioral process from a collection of space points that carry energy and quantum field attributes. The paper is considered by the author to represent a fairly complete causal model, except for some processes whose detailed behaviour needs to be determined by use of further computer simulations.
\end{abstract}

\section{Subject Areas}

Classical Physics, Special Theory of Relativity

\section{Keywords}

Spacetime Models, Discrete Spacetime, Relativity Theory, Causal Models, 
Quantum Field Theory, Quantum Loops, Bohmian Mechanics

\section{Introduction}

The author's work on causal models of quantum theory (QT), quantum field theory (QFT) and spacetime dynamics started with the attempt to develop a computer model of QT. Soon, the feasibility of such a QT computer model is impeded, not (as expected) by the strange and mysterious nature of QT, but by the many ambiguous formulations of the theory. The problems encountered (described in [1], [2] and [3]) lead to the conclusion that the apparent deficiencies of QT could (only?) be removed by the provision of a causal model of QT (including quantum field theory) and that the feasibility of constructing a causal model may be a criterion for the completeness of a physical theory in general.

The attempt to construct a local causal model of quantum theory, including QFT resulted in several refinement steps of the model (see Figure 1). At one point, it was recognized that a causal model of the dynamics of QT/QFT should better be based on a causal model of the dynamics of spacetime. Thus, a causal model of the dynamics of spacetime has been developed with these major goals: 1) as much as possible, be compatible with general relativity theory (GRT) and 2) should match the main features of the evolving model of QT/QFT. The causal model of spacetime dynamics is described in [4]. Because the model of spacetime dynamics is a major prerequisite of the work described in the present paper, a short description is also given in Section 4. In Section 5, the model of spacetime dynamics is applied to quantum fields and quantum objects. A bottom-up approach is taken

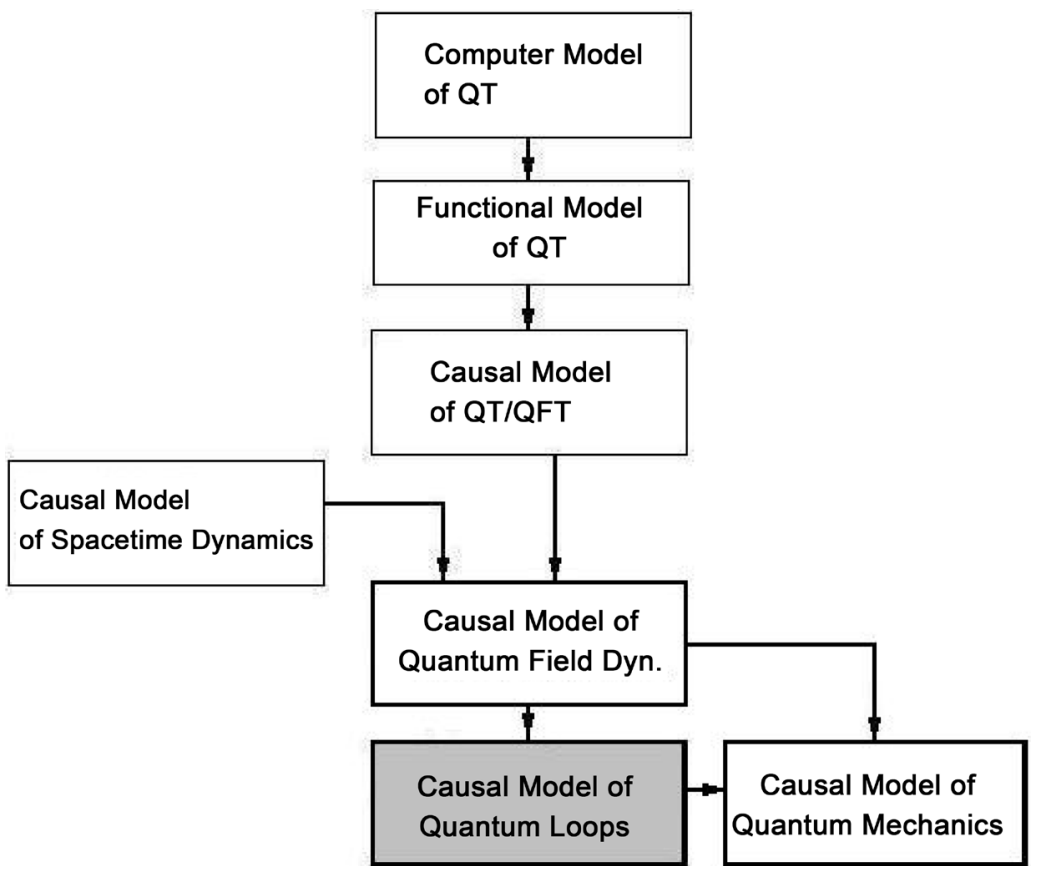

Figure 1. Refinement steps towards the causal model of quantum loops. 
here to a description of the causal model of QT/QFT dynamics. First, we describe how the model of spacetime dynamics is applied to quantum fields (Section 6), and we then examine how QFT processes result in the emergence of quantum objects (Section 7). Quantum loops and quantum loop process form the primary topic of this article, and play a major role in the emergence of quantum objects. After a description of the model for the establishment of quantum objects, Section 8 presents a discussion of quantum mechanics in terms of the model described in Section 7. In this model, in addition to generating quantum objects, quantum loops evaporate two types of "field": (i) the gravitational field (i.e., space curvature changes); and (ii) the Schrödinger field. The latter represents the wave function in the interpretation similar to the de Broglie-Bohm theory. The model of quantum mechanics presented in Section 8 therefore has some commonalities with the de Broglie-Bohm theory.

The author's work on models of QT/QFT and spacetime dynamics has been guided by three principles for models of physics theories, and the author has become increasingly convinced of these:

1) Causal models - the perception that it takes a causal model to explain the outcome of a physics experiment, and a complete causal model to explain the experimental results for a theory as far as possible.

2) Local causal models-not relying on (spooky) actions at a distance.

3) Discreteness of the essential parameters-the assumption that there exists a minimal size and granularity for the essential parameters of the theory.

Interestingly, the above principles also seem to be highly valued within the major modern physics theories, i.e., relativity and quantum theory. However, these two theories support different subsets of these three principles; relativity theory emphasises local causality, while quantum theory is founded on the discreteness (i.e. quanta) of energy. It is possible that the integration of the two theories requires adherence to all three principles.

Note that the specification of a model in the form of a local causal model is not just another style of description language. The description language used in this article is a consequence of very rigid requirements with respect to the required or allowed contents of the specification of a local causal model. This is described in more detail in Sections 2 and 3. Adherence to the three requirements of a local causal model, support for the discreteness of the essential model parameters and the need to describe nonlinear processes have resulted in a style of writing that may be considered by the reader to be not quite conformal with the style in which professional physics articles are typically written.

\section{Causal Models}

The formal definition of a (local) causal model has been published in various preceding papers from the author. It is here repeated because it is important to the subject of this paper and because an extended and refined treatment of local causal models is appropriate (see Section 3). 
Definition 1. The specification of a causal model of a theory of physics consists of 1) the specification of the system state, 2) the specification of the laws of physics that define the possible state transitions when applied to the system state, and 3) the assumption of a physics engine.

The physics engine. The physics engine represents the overall causal semantics of causal models. It acts upon the state of the physical system. The physics engine continuously determines new states in uniform time steps. For the formal definition of a causal model of a physical theory, a continuous repeated invocation of the physics engine is assumed to realize the progression of the system state.

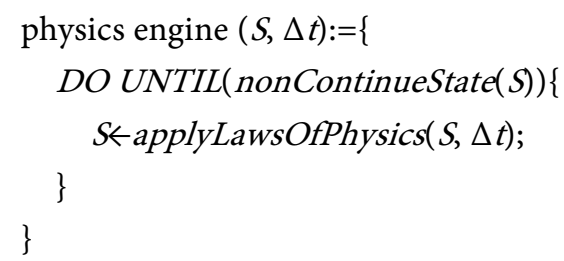

The system state. The system state defines the components, objects and parameters of the theory of physics that can be referenced and manipulated by the causal model. In contrast to the physics engine, the structure and content of the system state are specific for the causal model that is being specified. Therefore, the following is only an example of a possible system state specification.

$$
\begin{aligned}
& \text { systemstate }:=\{\text { spacepoint } \cdots\} \\
& \text { spacepoint }:=\left\{x_{1}, x_{2}, x_{3}, \psi\right\} \\
& \psi:=\left\{\text { stateParameter }_{1}, \cdots, \text { stateParameter }_{n}\right\}
\end{aligned}
$$

The laws of physics. The refinement of the statement

$S \leftarrow$ applyLawsOfPhysics $(S, \Delta t)$; defines how an "in" state s evolves into an "out" state s.

$$
\begin{aligned}
& L_{1}:=I F c_{1}(s) \text { THEN } s \leftarrow f_{1}(s) ; \\
& L_{2}:=I F c_{2}(s) \text { THEN } s \leftarrow f_{2}(s) ; \\
& \ldots \\
& L_{n}:=I F c_{n}(s) \text { THEN } s \leftarrow f_{n}(s) ;
\end{aligned}
$$

The "in" conditions $c_{i}(s)$ specify the applicability of the state transition function $f_{i}(s)$ in basic formal (e.g., mathematical) terms or refer to complex conditions that then have to be refined within the formal definition.

The state transition function $f_{i}(s)$ specifies the update of the state $\mathrm{s}$ in basic formal (e.g., mathematical) terms or refers to complex functions that then have to be refined within the formal definition.

The set of laws $L_{1}, \cdots, L_{n}$ has to be complete, consistent and conforming to reality (see [3] for more details).

In addition to the above-described basic forms of specification of the laws of physics by $L_{n}:=I F c_{n}(s) T H E N s \leftarrow f_{n}(s)$, other forms are also imaginable and sometimes used in this article. (This article does not contain a proper definition of the used causal model specification language. The language used is as- 
sumed to be largely self-explanatory.)

\section{Local Causal Models}

A local causal model is a special type of causal model. The subject locality and local causal model concern both, the system state and the laws of physics.

Spatial causal model A causal model of a theory of physics is called a spatial causal model if 1 ) the system state contains a component that represents a space, and 2) all other components of the system state can be mapped to the space. Many textbooks on physics (mostly in the context of relativity theory) and mathematics define the essential features of a "space". For the purpose of the present article, a more detailed discussion is not required. For the purpose of this article and the subject locality, it is sufficient to request that the space (assumed with a spatial model) supports the notions of position, distance and neighborhood.

Local causal model The definition of a local causal model presupposes a spatially causal model (see above). A (spatially) causal model is understood to be a local model if changes in the state of the system depend on the local state only and affect the local state only. The local state changes can propagate to neighboring locations. The propagation of the state changes to distant locations; however, they must always be accomplished through a series of state changes to neighboring locations. Special relativity requests that the series of state changes does not occur with a speed that is faster than the speed of light. This requirement is not considered essential for a local causal model.

Based on the formal model definition of a causal model, a formal definition of locality can be given.

Definition 2. A physical theory and a related spatially causal model are given. A causal model is called a local causal model if each of the laws $L_{i}$ applies to no more than a single position and/or to the neighborhood of this position.

The position reference can be explicit or implicit by reference to a state component that has a well-defined position in space.

Local spatial specifications If the causal model includes a model of spacetime dynamics (such as the model described in the present article), spatial specifications in the system state must not refer to globally (i.e., non-locally) arranged position, distance and direction specifications. This requirement, which is sometimes referred to as "background independence", prohibits references in terms of globally defined coordinate systems. An example where this requirement applies is Definition 4 in Section 4 containing direction specifications.

Physical Objects. Definition (2) notes a relatively strong type of locality that may be called "space-point locality". Most physics theories and models of physics theories contain spatially extended objects (e.g., particles, nuclei, stars, galaxies), with state components and attributes (such as mass, energy, momentum) that apply to the object as a whole. Causal model references to the complete space of a spatially extended object or to a property of the complete object are 
considered to violate locality. The construction of a local causal model may not be feasible. The space point locality and the feasibility of a local causal model may be regained, if it is possible to provide a model of the emergence of the object, in particular the emergence of object-global components and attributes. For example, in [5], the emergence of a quantum object is described as a collective behavioral process.

Proposition 1. Local causal models that include objects with (object-) global components and attributes are feasible only, if it is possible to show a model of the emergence of the (object-) global components and attributes.

The emergence of the object-global components and attributes is accompanied by the emergence of the object. (In general, it is possible to equate the emergence of the object-global components and attributes with the emergence of the object). Two typical ways/processes for the emergence of object-global components and attributes are

1) Aggregation of subcomponent attributes (example: aggregation of the mass of a physical object)

2) Synchronization of subcomponents attributes (examples: paths, velocity, momentum, angular momentum of a composite quantum object)

In Section 7, the model of the emergence of a quantum object is described as a collective behavioral process.

Global/local laws of physics. The provision of a local causal model may also be impeded by the existence of (object-) global laws of physics. Global laws of physics are laws that apply to a complete object. From the definition of a causal model given in Section 2, this means that the respective law of physics $L_{i}$ refers to some (object-) global components or attributes. Examples of global laws of physics are all kinds of conservation laws (e.g., energy conservation, momentum conservation), and the second law of thermodynamics (i.e., entropy law). In addition, the laws of quantum theory represent object-global laws, because the wave function may apply to a collection of particles. ${ }^{1}$ The existence of global laws within a theory of physics must not necessarily mean the non-feasibility of a local causal model, because the causal model may not include the global law within the relevant list $L_{1}, L_{2}, \cdots, L_{n}$ of the causal model. For example, the entropy law should not appear within a causal model. Neither should the global conservation laws appear in a causal model. The global conservation laws have to be broken down to (space-point) local conservation laws (which means the local laws have to obey the well-known symmetry requirements).

Even for global laws of physics that cannot be broken down to local laws, there may be ways to construct a local causal model. Because (as described above) a global law implies that there must be global object components and attributes, the feasibility of a local causal model may be regained, if it is possible to provide a model of the emergence of the object, in particular the emergence of object-global components and attributes.

${ }^{1}$ The object global nature of the wave function $\psi$ represents the root of the apparent non-feasibility of a local causal model of QT. 


\section{The Local Causal Model of Spacetime Dynamics}

\subsection{The Elementary Structure of Space(-Time)}

In the model described in this article, the system state consists of the space, fields and quantum objects. (Time is not considered part of the system state (see below "The space-time relationship")).

Definition 3. System state:=

Space,

Fields,

Quantum objects;

Definition 4. Space: $=\{$ spacepoint... $\}$;

spacepoint: $=\{\psi$, gravitationspec, connections $\} ;$

connections: $=\left\{\right.$ connection $_{1}, \ldots$, connection $\left._{n}\right\}$;

connection: $=\{$ neighborspacepoint, direction $\}$;

gravitationspec: $=\{$ gravitationdynamic, direction, gravitationstrength $\} ;$

$\psi$ represents the contents of space in the form of fields and quantum objects (see Section 5 for more details). According to Section 3, direction has to be a local parameter. As described in Section 3 "Local spatial specifications", to enable a local causal model, the direction specification of the connection must be given in terms of space-point-local parameters. In Section 6.4, a possible direction specification schema is described.

\subsection{The Space-Time Relationship}

In GRT and SRT, space and time are said to be integrated into spacetime. From a mathematical perspective, the integration of space and time is reflected in the use of vectors, matrices and tensors that combine the dimensions of space with that of time. The integration is also reflected in the laws of physics, where space and time (and their derivatives) are jointly transformed. As described above, in the causal model chosen here, space and time are strictly separated. Since this model also aims for maximal compatibility with GRT, the question arises of how this compatibility can be achieved with a model in which space and time are fundamentally (initially) not integrated. In the concept underlying the causal model of spacetime dynamics, space-time integration does not apply to space and time in general, as in SRT and GRT; instead, space-time integration only applies to physical processes executed in space and time.

This implies the following:

Assertion 2 The measure and metric for space and time can only be defined jointly for both space and time, and only with reference to a specific process that produces a specific rate of spatial change (i.e. length) within a specific time interval.

The physical process that is best suited for this joint definition of the measure for space and time is the movement of light, under the assumption that the speed of light is a constant.

Assertion 3 The execution speed of physical processes in terms of changes in 


\section{length in relation to the execution time is invariant.}

For example, if a clock rate (i.e., the proper time) changes, this is always accompanied by a length dilation in the space where the process is executed.

The major physical expressions of curved spacetime are length and time dilations. " "Time dilation" essentially means a dilation of the speed by which physical processes, such as clocks, run.

As a special case of Assertions 2 and 3:

Assertion 4 Length and time dilations are interrelated and occur only in combination.

Assertions 2 and 3 are essential in the more detailed model of spacetime dynamics described below. The above basic assertions with respect to the space-time relationship lead to the following propositions concerning the elementary structure of spacetime:

Proposition 5. The state update time interval, suti is a constant of nature.

Proposition 6. The distance between two neighboring space points, $l_{\text {connection }}$, is a constant of nature. This is the distance through which light moves during a suti.

(In Euclidean geometry, it is difficult to imagine that all space point connections have the same length if the connections are not restricted to orthogonal directions.)

In a model that assumes a constant speed of light, $c$, it follows from Propositions 5 and 6 that:

Proposition 7. During a state update time interval, suti, light moves a constant distance, namely the distance

$$
l_{\text {suti }}=l_{\text {connection }}=\operatorname{suti} \cdot \mathrm{c}
$$

The proposed model of spacetime dynamics assumes that all distances and lengths in space are composed of the elementary length units, $l_{\text {suti }}$. Likewise, all time intervals are multiples of suti. Lengths and distances are defined only between two space points and only with reference to the speed of light, c.

Proposition 8. The distance between two space points, $s p_{1}$ and $s p_{2}$ is given by the number of spacepoints, $n s p\left(s p_{1}, s p_{2}\right)$ through which light passes when moving from $s p_{1}$ to $s p_{2}$ multiplied by the elementary length unit, $l_{\text {suti }}$ $\left(=l_{\text {connection }}\right)$.

$$
\operatorname{distance}\left(s p_{1}, s p_{2}\right)=n s p\left(s p_{1}, s p_{2}\right) \cdot l_{\text {suti }} \text {. }
$$

The above propositions result in a model of spacetime in which the speed of light is a constant. However, due to Proposition 5, it is hard to avoid curved space. This does not present a problem, since curved spacetime is not undesirable in a spacetime model aiming for compatibility with GRT. The remaining problem is that of how to achieve GRT-compatible space curvature. Spacetime curvature due to time dilation (as predicted by GRT) also needs to be supported. The solutions offered by Assertion 3 and Propositions $4-7$ are that (i) the

${ }^{2}$ Throughout this article the term "dilation" is used to mean positive or negative dilation. 
process of space emergence/expansion (Section 4) results in length dilations through the suitable arrangement of space points and that (ii) length dilations cause clock rate dilations for processes running at space positions with dilated lengths.

The formal expression of point (i) is:

Proposition 9. Lengths within the gravitational field are dilated by the factor $F_{1}$.

The precise equation for the factor, $F_{1}$, such that it is in accordance with GRT is given below. For the model described in this article, the revised formulation of Assertion 4 is:

Proposition 10. Physical processes run faster or slower depending on the length dilation at the position in which the respective physical process is executed.

Proposition 9 may be viewed as a refinement of Assertion 3 above where the dilation of the clock rate concerns physical processes rather than the structure of spacetime. The major process that demonstrates the fixed relationship between the length dilation and the rate of change of the process is the propagation of light. This (simple) process is used as a measure for the change rate of other processes by setting the speed of light to be a constant, c. The next classes of processes in which the rate of change depends on the length dilation in precisely the same proportions as in the propagation of light are clocks in differing realisations. In summary, there is no direct reflection of time dilation as an attribute of spacetime in the model of spacetime dynamics. Clock rate dilation (rather than time dilation) arises as a property of processes running within space. The clock rate dilation factor can be derived from the length dilation factor, $F_{1}$ of the space points at which the respective process is currently being executed. Thus, in the model of spacetime dynamics, two levels of time are distinguished, although these are seen as a single entity in GRT/SRT:

1) At the basic level, the progression of time is determined by the uniform state update time interval, suti. Simultaneity is assumed for all state changes occurring within the same state update cycle.

2) Differing clock rates, proper times, and the relativity of simultaneity are not associated with the basic overall spacetime (level 1), but instead are associated with physical processes running in space.

In terms of space, two levels can also be distinguished, although these are two levels of consideration:

- At the abstract level (the mathematical level), the space consists of a set of interconnected space points. The issue of whether or not the totality of the interconnected space points represents a Euclidean space or a specific topology (e.g., a Riemann manifold) is left open.

- At the physical level (the essential level), physical meaning is assigned to the components of the space point and its connections. In particular, the length of the connections is no longer a geometrical property, but specifies only the 
$\Delta$ length through which light moves during the state update time interval, suti.

Thus, the integration of space and time into spacetime is established in the model of spacetime dynamics by the physical meaning assigned to the components of the space points and their connections.

The length dilation factor $F_{1}$. In GRT, the curvature specification (i.e., the curvature tensor), contains a time-related component in addition to the three space-related components. As an example of the impact of the time factor, the gravitational redshift is explained as the consequence of the time factor in the spacetime curvature (see, for example, [6], page 231). With a Schwarzschild metric

$$
\Delta s^{2}=-\left(1-\frac{2 G M}{c^{2} r}\right)(c \Delta t)^{2}+(\Delta x)^{2}+(\Delta y)^{2}+(\Delta z)^{2}
$$

This means that a clock at position $(x, y, z)$ would run slower than a clock that is not affected by a gravitational field by a factor

$$
F_{1}=\sqrt{1-\frac{2 G M}{c^{2} r}}
$$

A standard clock at some point A of low potential (for example, at the surface of the earth) would run slower than the same clock at a point B with higher potential (for example, in a GPS satellite). Proposition 8 states that not only are the clock rates of clocks within a gravitational field dilated by the factor $F_{1}$, but that this dilation also applies to lengths. (As a supporting argument, only in this way can the assertion of the constant speed of light be maintained.) Proposition 9 also means that length dilation is the primary effect, and that the clock rate dilation for clocks residing in the length-dilated space is a consequence of the length dilation.

\section{Energy dilation with objects moving in curved space}

Proposition 11. When an object (e.g. a particle) moves from one space point, $s p_{1}$, to another, $s p_{2}$ the energy of the object decreases or increases as a function of the difference in the gravitationstrength of the two space points.

The energy difference associated with $s p_{1}$ and $s p_{2}$ is usually called the (difference in) potential energy of the positions of $s p_{1}$ and $s p_{2}$. The gravitationstrength and thus the energy, increases or decreases and has a direction, which is towards the source(s) of the gravitation. The basic types of energy that are affected by the increase or decrease in the positional energy are the kinetic energy and the wave energy (i.e., the wave frequency and wavelength). Appendix $C$ shows a summary of the time, length and energy dilations for standard GRT and for the causal model of spacetime dynamics.

\subsection{The Dynamics of Space Emergence and Space Changes}

With the proposed model, it is assumed that all the dynamics of space changes, including the emergence of space, starting from a minimal source and proceeding through the successive addition of new surface layers of space points. The number of space points at the surface layer increases with each new surface 
layer. The space expansion factor (implied by the space expansion algorithm) determines the increase of the number of space points with the new surface layer. Figure 2 shows examples of space surface layers with different expansion factors $(2$ or 3$) .{ }^{3}$.

The space expansion algorithm must achieve compatibility with GRT. This affects two items:

1) The expansion factor that determines the growth of the number of space points at the surface layers must be such that Equation (2) is satisfied (which means that a Schwarzschild metric arises).

2) The gravitationstrength in Definition 4 that specifies the strength of the gravitational field $\psi_{G}$ must decrease with the increasing distance from the source of the gravitation (see parameter $r$ in Equation (2)). If multiple sources are in the process of aggregation (see below), the strength of the gravitational field has to increase accordingly.

The process of space state progression consists of the repeated application of Spread-out/Bundle operations to all space points that are identified by gravitation dynamic $\neq 0$ (see Definition 4).

Specification 5 Space-state-progression ( space $s p$ ): $=\{$

Spread-out: FOR (ALL spacepoints sp.point [i]) \{

IF (sp.point[i].gravitationdynamic $\neq 0$ ) \{

generate-OUT-points-from(sp.point $[i]$ );

\}

Bundle: FOR (ALL new spacepoints sp.point $[j])\{$ accumulate-inconnections (sp.point $[j]$ ); \}

The expression "generate-OUT-points-from()" generates new space points (including the necessary connections) for all space points that are currently with

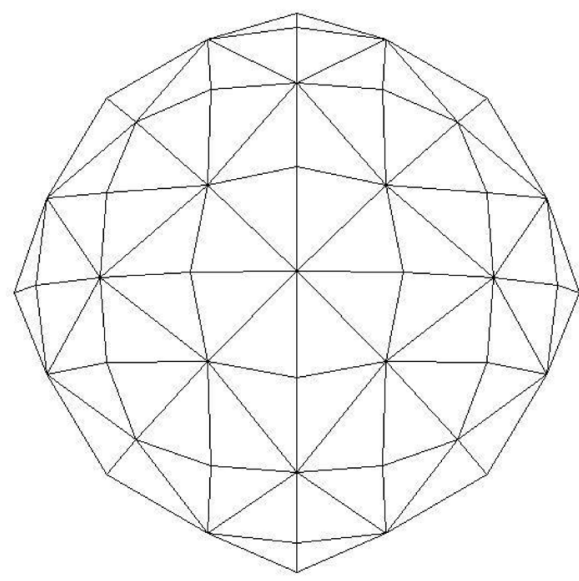

expansion factor $=2$

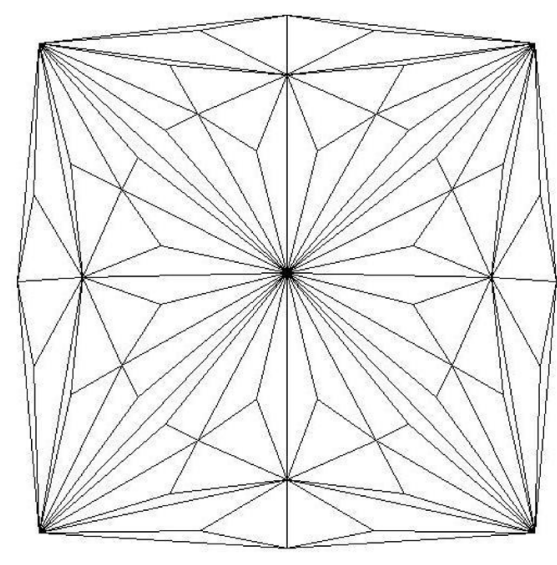

expansion factor $=3$

Figure 2. Surface of small space objects with radius $=3 \cdot l_{\text {suti }}$.

${ }^{3}$ Remember that we are dealing with curved space and that this cannot be adequately represented in the 2-dimensional figure. 
this attribution: gravitation dynamic $\neq 0$. At least one new space point is generated. Whether further space points and connections emerge depend on the gravitational strength and on the more detailed algorithm for the emergence of space changes. The gravitational strength is reduced as a function of the increasing distance from the gravitational source(s). The new space points are temporarily marked as NEW in the "Bundle" step, in which the gravitational strengths are accumulated for all connections to the new space point. The function "generate-OUT-points-from" can be expressed by the following specification.

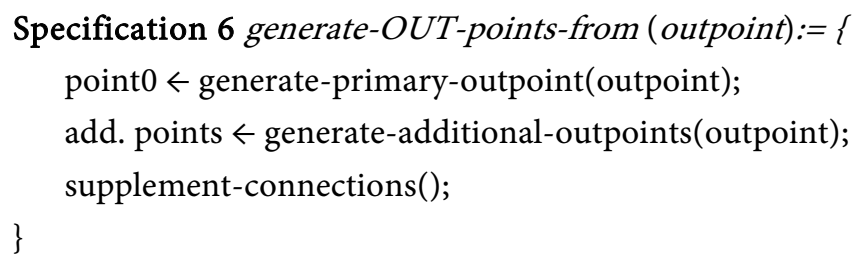

Aggregation of space changes from multiple sources. The assumption that all space change dynamics starts from minimal sources implies that the space changes originating from multiple sources, typically, will soon start to overlap and will accumulate. This results in an overall process of space dynamics where three phases can be distinguished:

- Phase 1: The space changes from the individual sources propagate by the addition of spherical surface layers as described above. The changed space object represents a Schwarzschild metric and Equation (2) is satisfied.

- Phase 2: The space changes overlap and have to be accumulated. The accumulated space object does not represent a Schwarzschild metric and Equation (2) is not applicable.

- Phase 3: At a suitable distance from the gravitational sources, the gravitation may be handled as if there was a single source with the mass equal to the sum of the multiple sources of masses located at the centre of masses. Equation (2) is applicable again.

Further details on the subject of Section 4 can be found in [7] and [4].

Sources of space change dynamics. In [7] and [4], the sources of space change dynamics are described as quantum objects. In the present article, the model of spacetime dynamics is also applied to the dynamics of quantum objects and quantum fields. This leads to a refinement of the model in which the elementary processes within the quantum objects are already sources of space change dynamics (see Section 6.1).

\section{Application of the Model of Spacetime Dynamics to QT/QFT}

The local causal model of spacetime dynamics described in [7] and [4] and summarized in Section 4 has been developed with the goal of providing a basis for a local causal model of QT/QFT. The application of the model to quantum theory and quantum field theory is described in the following: 


\subsection{The Space Contents}

Definition 3 defines the system state of the local causal model of spacetime dynamics as consisting of space, fields and quantum objects. Fields and quantum objects may be viewed as the contents of space. In Definition 4, the space point is defined as containing the component $\psi . \Psi$ is said to represent the point-local content of the space. Application of the model of spacetime dynamics to QT/QFT requires 1) a more detailed specification of the space contents $\psi$ and 2) the specification of the model of the dynamics of the space contents.

The space point component $\psi$ may represent different types of space contents, that is, different types of fields and of particles. The possible types of $\psi$ contain the field types known from QFT (e.g., the electromagnetic field), the gravitational field $\psi_{G}^{4}$ and the "Schrödinger field" $\psi_{S}$ that represents the wave function. ${ }^{5}$ The association of $\psi$ to the space point is not a static association. The space point content may move and spread out to neighboring space points. Also, the $\psi$ of a common type may form collections such as fields, particles and composite quantum objects. Such collections of $\psi$ may emerge to physical objects (i.e., quantum objects) that propagate as an entity with special object-global attributes.

The more detailed components of $\psi$ depend on the field type. A fairly general set of components and attributes is

Definition 7. $\psi:=\{$

dynamics attributes,

spin type, spin value,

charge type, charge value,

direction

\}

dynamics attributes: $=\{$ amplitude, frequency $\}$

Fields are the simplest type of $\psi$ collections

$$
\text { field }:=\left\{\text { spacepoint }_{1} \cdot \psi, \text { spacepoint }_{2} \cdot \psi, \cdots\right\} .
$$

All the space points belonging to the field have the same field type associated. In contrast to quantum objects, there are no object-global attributes associated with the field. This makes the fields a suitable base for the specification of a (space point) local causal model of QT/QFT.

A quantum object is defined as consisting of a collection of 1 to $\mathrm{n}$ particles (see [8]). This means that a quantum object is either an elementary particle or a composite quantum object.

Definition 8. quantumobject: $=\{$

globalquantumobjectattributes $\Omega$;

particle,

$\cdots$

particle $_{n}$

\}

${ }^{4}$ In Definition 4, the gravitational field $\psi_{G}$ is represented by the gravitationspec attribute.

${ }^{5}$ The relation between the Schrödinger field $\psi_{S}$ and the QFT fields will be discussed in Sections 7 and 8. 
The collection of particles is supplemented by global attributes $\Omega_{1}, \Omega_{2}, \cdots, \Omega_{j}$.

The elementary particle encompasses the $\psi$-components of a set of space points and global particle attributes $\Theta_{1}, \Theta_{2}, \cdots, \Theta_{j}$.

Definition 9. particle: $=\{$

globalparticleattributes $\Theta$;

spacepoint $_{1}$, spacepoint sp.... $_{2}$.

\}

Examples of global attributes are $\Omega_{\text {mass }}, \Omega_{\text {charge }}$ and $\Omega_{\text {spin }}$. As described in Section 3, the occurrence of global attributes in a local causal model may disturb the (space point) locality of the model, if it is not possible to show the emergence of the global attribute from (space point) local parameters.

\subsection{Space Contents Dynamics}

The model of the dynamics of space contents (quantum fields and quantum objects) is formulated using a bottom-up approach. The basis of the causal model of QT/QFT is the local causal model of quantum fields (Section 6), an extension of the local causal model of spacetime dynamics described in [7] and [4] and summarised in Section 4. Quantum objects emerge from quantum fields. In this way, the model distinguishes the emergence of elementary particles and the dynamics of composite quantum objects. Elementary particles emerge directly from quantum fields in a collective behaviour process called a quantum loop (Section 7). The dynamics of the complete quantum mechanics, including composite quantum objects, is briefly addressed in Section 8.

\section{Quantum Field Dynamics}

\subsection{Energy-Carrying Space Points-The Sources of Quantum Field Dynamics and Spacetime Dynamics}

In [7] and [4] and in Section 4.3, quantum objects are denoted as the sources of spacetime dynamics. With the application of the model of spacetime dynamics to QT/QFT, quantum objects remain a source of spacetime dynamics; however, the model is refined to include, in addition, specific space points as the source of spacetime dynamics and as the sources of quantum field dynamics. The space points that are sources of spacetime dynamics may be called energy-carrying space points. The contents of energy-carrying space points propagate through space. In the formal specification of the QT/QFT model, energy-carrying space points contain non-empty dynamics attributes as part of the field contents $\psi$ (see Definition 7). A space point $s p_{i}$ is never a permanent source of quantum field dynamics. After the propagation of the contents of $s p_{i}$ has taken place, the dynamics attributes are reset to zero.

In addition to the dynamics attributes (indicating that the space point is an actual source of quantum field dynamics), the propagation direction is specified as an additional attribute. According to Definition 7 , the fields represented by $\psi$ may have different spin values. In the model described here, fields may have spin 
1/2 (a fermionic field) or spin 1 (a bosonic field). In addition to the classical field types, two (secondary) types of fields are generated in the dynamics of energy-carrying space points:

1) Gravitational fields, $\psi_{G}$ : The dynamics of space changes (i.e., of gravitation) was the starting point for the causal model of quantum field dynamics. The integrated model of spacetime and quantum field dynamics considers gravitation as a special type of field.

2) Schrödinger-fields, $-\psi_{s}$ : The wave function of quantum mechanics requires a representation at the space (point) content and a causal model of its dynamics. In the model described here, this "field" is called the "Schrödinger-field" $-\psi_{s}$.

These two types of field are considered to be secondary fields, since they do not carry energy, meaning that: 1) the creation of these types of fields due to the propagation of energy-carrying space points does not reduce the energy of the source; and 2) the fields $\psi_{G}$ and $\psi_{S}$ are not capable of interacting with other quantum fields and quantum objects by exchanging energy. Unlike primary fields, the propagation of secondary fields does not need to preserve the direction of momentum. This enables the expansion of these fields to create/cover an ever-growing volume of space.

\subsection{Quantum Fields Are Waves}

Waves and fields are the basic constituents of quantum field theory. Since we are dealing here with the lowest level of space granularity, it is difficult to imagine the application of the classical model of waves to the model of quantum field dynamics. Nevertheless, there are a number of properties that are known from the physics of waves that also appear to be useful in the model of quantum field dynamics presented here. A very short introduction to waves in physics is therefore given in the following. The description below is derived from [9] and to a larger extent from [10].

The standard formulation of the "wave equation", that is, the equation of motion for waves, is (see, for example [9])

$$
\left(\frac{1}{v^{2}} \frac{\partial^{2}}{\partial t^{2}}-\nabla^{2}\right) \psi(x, t)=0
$$

Depending on the particular context, this equation may be varied or extended by setting the right-hand side not equal to zero. For example, in [10], two classes of waves, Class 0 and Class 1, are distinguished:

Class 0:

$$
\mathrm{d}^{2} \psi / \mathrm{d} t^{2}-c_{w}^{2} \mathrm{~d}^{2} \psi / \mathrm{d} x^{2}=0
$$

Class 1:

$$
\mathrm{d}^{2} \psi / \mathrm{d} t^{2}-c_{w}^{2} \mathrm{~d}^{2} \psi / \mathrm{d} x^{2}=-\left(2 \pi v_{\min }\right)^{2}\left(\psi-\psi_{0}\right) .
$$

For the mapping of QFT to the causal model of QT/QFT, the way in which the energy of a wave is reflected in the wave equations is important. In [10], the 
quantum waves are described by a motion formula

$$
Z(x, t)=Z_{0}+A \cos (2 \pi[v t-x / \lambda])
$$

where $\mathrm{A}$ is the amplitude, $\lambda$ is the wavelength and $v$ is the frequency. In quantum mechanics, the amplitude $\mathrm{A}$ is restricted to discrete values. The equation of motion requires that the frequency and wavelength be related to $v_{\min }$ (appearing in Equation (6)) by the formula

$$
E^{2}=(h v)^{2}=(h c / \lambda)^{2}+\left(h v_{\min }\right)^{2}
$$

This looks like a Pythagorean relation (i.e., $c^{2}=a^{2}+b^{2}$ ). Another Pythagorean relation, well-known in relativity theory, describes the relation between total energy $E$, the kinetic energy $p \cdot m$ and the mass energy $m c^{2}$ by

$$
E^{2}=(p c)^{2}+\left(m c^{2}\right)^{2}
$$

This suggests the following equation for the total energy, the kinetic energy and the mass energy:

$$
E=h v ; p c=h c / \lambda ; m c^{2}=h v_{\min } .
$$

A (simple) wave of a given frequency and wavelength is made up of $n$ quanta. The allowed values of the amplitude $A$ are proportional to $\sqrt{n}$. For bosons, the allowed values of the energy are

$$
E=(n+1 / 2) h v, \text { where } n=0,1,2,3,4 .
$$

For fermions the allowed values are

$$
E=(n-1 / 2) h v \text {, where } n=0 \text { or } 1 .
$$

In addition to the above considerations, which relate to the propagation of a single wave, the physics of interacting waves offers another basis for a causal model of quantum field dynamics. In [10], an example of the interaction of quantum fields is described by three equations of motion in which the interacting waves occur on the right-hand side:

$$
\begin{gathered}
\mathrm{d}^{2} A / \mathrm{d} t^{2}-c^{2} \mathrm{~d}^{2} A / \mathrm{d} x^{2}=y B C . \\
\mathrm{d}^{2} B / \mathrm{d} t^{2}-c^{2} \mathrm{~d}^{2} B / \mathrm{d} x^{2}=y A C . \\
\mathrm{d}^{2} C / \mathrm{d} t^{2}-c^{2} \mathrm{~d}^{2} C / \mathrm{d} x^{2}=\left(2 \pi v_{\text {min }}\right)^{2} C+y A B .
\end{gathered}
$$

Depending on the specific attributes of the interacting waves, other expressions describing the results of this interaction are also possible, and the example given in [10] is

$$
\begin{gathered}
\mathrm{d}^{2} S / \mathrm{d} t^{2}-c^{2} \mathrm{~d}^{2} S / \mathrm{d} x^{2}=\left(2 \pi v_{\min }\right)^{2}\left(m_{S}^{2}[S-S 0]+y^{2} S Z^{2}\right) . \\
\mathrm{d}^{2} Z / \mathrm{d} t^{2}-c^{2} \mathrm{~d}^{2} Z / \mathrm{d} x^{2}=\left(2 \pi v_{\text {min }}\right)^{2} y^{2} S^{2} Z .
\end{gathered}
$$

Further rules for the determination of the results of interacting fields are given in QFT, in terms of the Feynman rules for particle scattering.

Although it does not appear to be reasonable to apply the classical model of 
waves in its entirety to the causal model of quantum field dynamics, the following items are taken over and mapped to this model:

- The energy of an energy-carrying space point is proportional to the amplitude and frequency parameters.

- The allowed values of the amplitude A are proportional to $\sqrt{n}$.

- For bosonic energy-carrying space points, the allowed values of the energy are $E=(n+1 / 2) h v$, where $n=0,1,2,3,4$.

- For fermionic energy-carrying space points, the allowed values are:

- $E=(n-1 / 2) h v$, where $n=0$ or 1 .

- There may be a minimal frequency $v_{\min }$.

- Equation (6) may also apply to the propagation of energy-carrying space points.

\subsection{The Interaction Operator $\chi$}

In the model of the propagation of space contents (fields and quantum objects), the space point connections are dynamically assigned to "in-connections" and "out-connections". Figure 3 shows a typical space point, with 14 connections that span the whole neighbourhood space. In the proposed causal model of QT/QFT, all space point connections may be utilised for the propagation of fields (i.e., gravitational, fermionic, bosonic, Schrödinger field). This enables support for the concurrent propagation of the different field types. All space point connections are utilised for the propagation of the gravitational field and the Schrödinger field, and all connections that are not in-connections become out-connections. In the propagation of fermionic and bosonic fields, the direction has to be preserved in the form of geodesic paths. This includes the possibility of the creation and annihilation of field types according to the rules of QFT (e.g. Feynman rules). Unlike in standard QFT, multiple bosonic field connections are possible. If more than one bosonic in-connection occurs (dynamically) at a space point, the bosonic in-connections are accumulated and treated like a single in-connection. A bosonic out-connection may be distributed to multiple out-connections.

The propagation of quantum fields through space is concentrated in the interaction operator $\chi \cdot \chi(s p)$ corresponds to combinations of creation and annihilation operators in QFT, and applies to a space point sp, including its dynamically

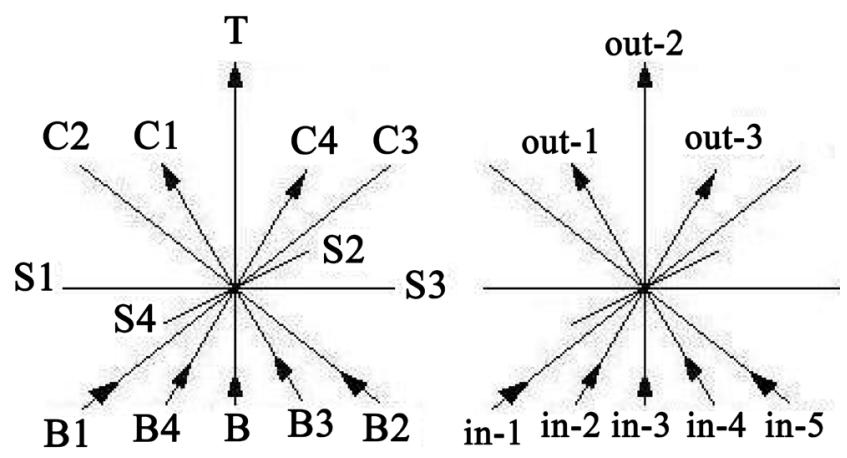

Figure 3. Example of the distribution of connections of a space point. 
assigned in-connection and out-connections. For a given space point, sp, it determines the out-connections and the new state (including contents) of the space points that are targets of the out-connections as a function of the content of sp. The overall field state progression can be expressed as:

Field-state-progression ( space): $=\{$

FOR (ALL spacepoints $s p[i])\{$

IF ( $s p[i]$. gravitationdynamic $\neq 0$ OR $s p[i] . \psi \cdot$ dynamicsattributes $\neq 0)$ apply $\chi(s p)$;

\}\}

\section{Specification 10 apply $\chi(s p):=\{$}

Spread-out:

determine-gravitational-out-connections $(s p)$;

distribute-gravitation $(s p)$;

distribute- $\psi_{s}(s p)$;

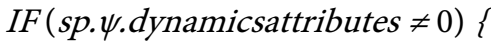

IF (sp. $\psi$.spintype $=1 / 2)$ distribute-fermion $(s p)$;

else distribute-boson $(s p)$;

\}

Bundle:

FOR (ALL spacepoints sp[i] WITH inconnections) \{

bundle-gravitational-inconnections(sp[i]);

bundle- $\psi_{s}$-inconnections(sp[i]);

bundle- $\psi$-inconnections(sp[i]);

set-dynamics-attributes(sp[i]);

\}

\}

Thus, the application of $\chi(s p)$ consists essentially of a combination of Spread-out and Bundle, where Spread-out determines the out-connections of sp and Bundle bundles all in-connections of those space points that have them (resulting from Spread-out). For the gravitational field $\psi_{\mathrm{g}}$ the combination of distribute-gravitation () and bundle-gravitational-inconnections results in the propagation of space (curvature) changes as described in Section 4.3. The Schrödinger-field $\psi_{S}$ is assumed to propagate in a similar way to the gravitational field, except that the Schrödinger equation in Equation (8) must be obeyed.

The combination of $\{$ distribute-fermion() and distribute-boson() $\}$ and bundle- $\psi$-inconnections() is more complicated, since the rules of QFT (e.g. the Feynman rules) must be satisfied. This means, for example, that distribute-fermion(sp) must not result in two fermionic out-connections of the same type and charge. In addition, in both Spread-out and Bundle, the energy and momentum must be preserved. The detailed determination of the momentum and energy of the out-connections is also derived from QFT, although with significant adaptations due to the characteristics of the causal model. The major differences from standard QFT are as follows: 
- The model of quantum field dynamics described here applies to energy-carrying space points, while the QFT rules for the calculation of scattering matrix amplitudes apply to (virtual) particles.

- Since this is a causal model, the amplitude associated with a propagation path must be an explicit system state parameter that has a non-probabilistic meaning. In the proposed model, the amplitude of an energy-carrying path is a measure of the energy of the energy-carrying space point (as opposed to a probability amplitude).

- There is no relativity of time progression (at the level of quantum field dynamics).

- Since the model is a local causal model, $\chi(s p)$ must depend only on space point-local parameters. This affects the parameter "mass", which occurs frequently in standard QFT for calculations of scattering matrices. Another implication is that the specification of spatial directions must be in terms of space point-local parameters (see below Section 6.4).

- Since a space point has only a small, discrete number of connections to neighbouring space points, only a small set of possible directions (or even a single definite direction) must be determined, where QFT applies integrals that span the total space.

- Distances between vertices have a fixed length (i.e., the length of space point connections are constant $=L_{\text {connection }}$, see Section 4.1).

- The possible energy and momentum values are quantised (i.e., discrete values with a non-zero minimum value).

\subsection{The Local Space-Point Specification of Directions}

The laws of physics require conservation of momentum when the field contents $\psi$ of fermionic or bosonic fields propagate from the in-connection(s) of a space point to the out-connection(s). Conservation of momentum means (besides conservation of the amount) conservation of the direction of the propagation. That is, the direction of the out-connection must be equal to the direction of the in-connection. For example, in Figure 3 the direction of in- 3 is equal to that of out-2. The requirement of direction conservation is a trivial requirement with non-curved (e.g. Euclidean) space with global direction specification in terms of a globally agreed coordinate system. With a local causal model according to Section 3 and curved discrete space, the implementation of the requirement is less trivial. As described in Section 3 "Local spatial specifications", to enable a local causal model, the direction specification of the connection must be given in terms of space-point-local parameters and the algorithm for the determination of out-connections must use only the local direction specification. A direction specification scheme that satisfies this requirement is (roughly) described in the following. To simplify the description, let us assume that the typical space point has 14 connections with the following labels and (local) meanings:

- B: The connection from the direction of the source (e.g. in-3 in Figure 3)

- $\mathrm{T}$ : The connection from the direction away from the source (e.g. out-2 in 
Figure 3)

- S1, S2, S3, S4: The connections that are orthogonal to B and T.

The connections $S_{i}$ are those connections between space points that have an equal distance from the gravitational source. In the model of the emergence of space described in Section 4, the emergence of space continuously develops surfaces such as the ones shown in Figure 2 that consist of $S_{i}$ connections ("S" stands for surface).

- B1, B2, B3, B4 The connections between B and S1, S2, S3, S4 (e.g., in-1, in-2, in-4, in-5 in Figure 3)

- C1, C2, C3, C4 The connections between T and S1, S2, S3, S4 (e.g., out-1, out-3 in Figure 3)

This direction specification scheme assumes that for each connection/direction an opposite connection/direction exists. If the set of connections is $\{\mathrm{B}, \mathrm{T}, \mathrm{S} 1, \mathrm{~S} 2, \mathrm{~S} 3, \mathrm{~S} 4, \mathrm{~B} 1, \mathrm{~B} 2, \mathrm{~B} 3, \mathrm{~B} 4, \mathrm{C} 1, \mathrm{C} 2, \mathrm{C} 3, \mathrm{C} 4\}$, the corresponding opposite connections are opposite $(\{\mathrm{B}, \mathrm{T}, \mathrm{S} 1, \mathrm{~S} 2, \mathrm{~S} 3, \mathrm{~S} 4, \mathrm{~B} 1, \mathrm{~B} 2, \mathrm{~B} 3, \mathrm{~B} 4, \mathrm{C} 1, \mathrm{C} 2, \mathrm{C} 3$, $\mathrm{C} 4\} \rightarrow\{\mathrm{T}, \mathrm{B}, \mathrm{S} 3, \mathrm{~S} 4, \mathrm{~S} 1, \mathrm{~S} 2, \mathrm{C} 3, \mathrm{C} 4, \mathrm{C} 1, \mathrm{C} 2, \mathrm{~B} 3, \mathrm{~B} 4, \mathrm{~B} 1, \mathrm{~B} 2\})$.

Based on the above direction specification scheme and the existence of the opposite() operator, it is possible to determine the out-connection for a given in-connection. ${ }^{6}$ The successive application of the scheme determines geodesic paths through discretized curved space. As a general observation, the assumption of discrete entities, such as discrete geodesic paths, may result in non-smooth effects at a very small scale.

Geodesics of quantum fields in the model of spacetime dynamics The geodesics of energy-carrying space points are determined by 1) the structure of spacetime, and 2) the algorithm that decides which out-connection(s) correspond to the given in-connection(s). In the simplest case, where a single out-connection is assigned to a single in-connection, the determination of the out-connection that corresponds to a given in-connection is straightforward. As described in the above space-point local specification scheme, for each possible in(out)-connection there exists an opposite out(in)-connection. Notice, however, that because we are dealing with curved discrete space, the "opposite" direction cannot be defined in the same way as in Euclidean space. Nor is it possible to define geodesics in the way they are defined with differentiable Riemannian manifolds. In a model of spacetime dynamics in curved discrete spacetime, direction conservation and geodesics must be defined in terms of space point-local parameters, that is, in terms of the discrete space-point connections. This may lead to geodesic paths that loop on the surface of an emerging space object (see Section 7, Quantum loops).

\section{Quantum Loops}

Proposition 12. Quantum objects, elementary particles as well as composite

${ }^{6}$ The assumption of 14 space point connections and the validity of the symmetric opposite() operator (i.e., opposite $(\operatorname{opposite}(\mathrm{c}))=\mathrm{c}$ ) are not generally satisfied with the proposed causal model. However, this makes the described algorithm only slightly more complicated and the results, a matter of statistics. 
quantum objects, are realized by quantum loops.

The assumptions that (1) space dynamics (e.g., the emergence of space and the propagation of space changes) starts already at the energy-carrying space points and that (2) a de facto strong space curvature near the minimal sources of space dynamics already exists, enable a causal model of the emergence of quantum objects. The collective behavioral process, called "quantum loop" emerges when a multitude of energy-carrying space points are confined in a small volume of curved space called the quantum loop shell.

Collective behavioral processes are characterized by the (loosely) synchronized behavior of a collection of elements of equal type. Prerequisites for the occurrence of collective behavior are (see, for example [5]):

- a multitude of elements of equal type,

- elements residing within a (small) volume of space such that interactions between the elements are enabled,

- interactions between the elements that lead to synchronizations with respect to specific properties.

Typically, external influences can support or destroy the collective behavior and phase transitions occur when the frequency and strength of the interactions increases or decreases due to collective energy increase/decrease. Collective behaviour is always the result of some process with possible phase transitions to stable states. These stable states may be called "quantum equilibrium" (see Section 8.4, Proposition 17 and [11] for more details on quantum equilibrium).

Major characteristics and parameters of a quantum loop. Quantum loops constitute quantum objects, elementary particles and composite quantum objects. In the present article, only the generation of elementary particles by quantum loops is discussed in detail (the generation of composite quantum objects is briefly discussed in Section 7.5). Quantum loops that form elementary particles have the following components and characteristics:

- The space occupied by the quantum loop contains $N_{s p}$ space points. Since the quantum object represented by the quantum loop is generally moving, the set of space points occupied by the quantum loop changes dynamically. In addition, the size of the quantum loop, $N_{s p}$, can vary dynamically.

- Of the $N_{s p}$ space points belonging to the quantum loop, $N_{e c}$ space points are energy-carrying space points $\left(N_{e c}<N_{s p}\right)$. As a consequence of the continuous interactions between the $N_{e c}$ energy-carrying space points, $N_{e c}$ also changes dynamically.

- The set of $N_{e c}$ energy-carrying space points has a major field type $\psi_{q l}$. Due to the continuous interactions between the energy-carrying space points, field types other than the major field type $\psi_{q l}$ may temporarily occur.

- The total energy of the quantum loop $E_{q l}$ is constant (if external influences are excluded).

- As a result of the collective behaviour process, the energies of the energy-carrying space points $E_{e c}$ will become (roughly) equal to $E_{e c}=E_{q l} / N_{e c}$. 
Conditions for the constitution of quantum loops. For the emergence of quantum loops and, more importantly, for the stable lifetime of an established quantum loop, two conditions must be satisfied:

1) The space within which the internal quantum loop dynamics is executed must have a curvature that enforces the confinement of the energy-carrying space points within the shell.

2) The quantum loop dynamics (i.e., the propagation and interactions of the energy-carrying space points) must preserve the energy of the total quantum loop, $E_{q l}$.

The first condition concerns the model of spacetime, and in particular its curvature (Section 4), while the second concerns the model of quantum field dynamics, and in particular the interaction operator $\chi$ (Section 6.3).

\subsection{The Gravitational Field $\psi_{G}$ around the Sources of Space Dynamics}

In Section 4, the model of the emergence of space and of the propagation of space changes is described as resulting in the development of successive layers of spherical surfaces, with a strong curvature near the source, i.e., the quantum object. The refinement of the model described in Section 6.1 assumes that the energy-carrying space points are already deeper sources of space curvature and gravitation. In this model of spacetime dynamics, space curvature is represented by two parameters:

1) Space curvature is represented by the density of these space points and by the arrangement of the connections between them. As described in Section 6.4, energy-carrying space points follow geodesic paths in space. If a geodesic path lies on a surface layer around the gravitational source (i.e., if the path moves from space point $s p_{1}$ to $s p_{1}$, where both space points are at equal distances from the source), the geodesic path continues at the surface layer, forming a "geodesic loop".

2) The gravitation field $\psi_{G}$ is assigned to each space point in form of the gravitationspec (see Definition 4). The variations in the gravitationstrength may be viewed as the establishment of space curvature. ${ }^{7}$

Both effects may contribute to the confinement of the quantum loop within the quantum loop shell. Scenarios can be imagined in which the collective behaviour of the energy-carrying space points results in a significant proportion of them ending up in a geodesic loop. Figure 4 shows the mapping of the surface of the space object shown in Figure 2 to a two-dimensional flat plan, and an example of a geodesic loop on that surface (the space curvature is not recognisable in both Figure 4 and Figure 2). With the chosen type of 2D mapping, one pole of the surface (e.g., the north pole P1) is shown at the centre, while the opposite pole (e.g., the south pole, P188) appears eight times. The bold path shown in Figure 4 represents a simple geodesic loop around the surface, which meets the

${ }^{7}$ GRT formulates this the other way around, i.e., the space curvature controls the variation in the gravitation strength. 


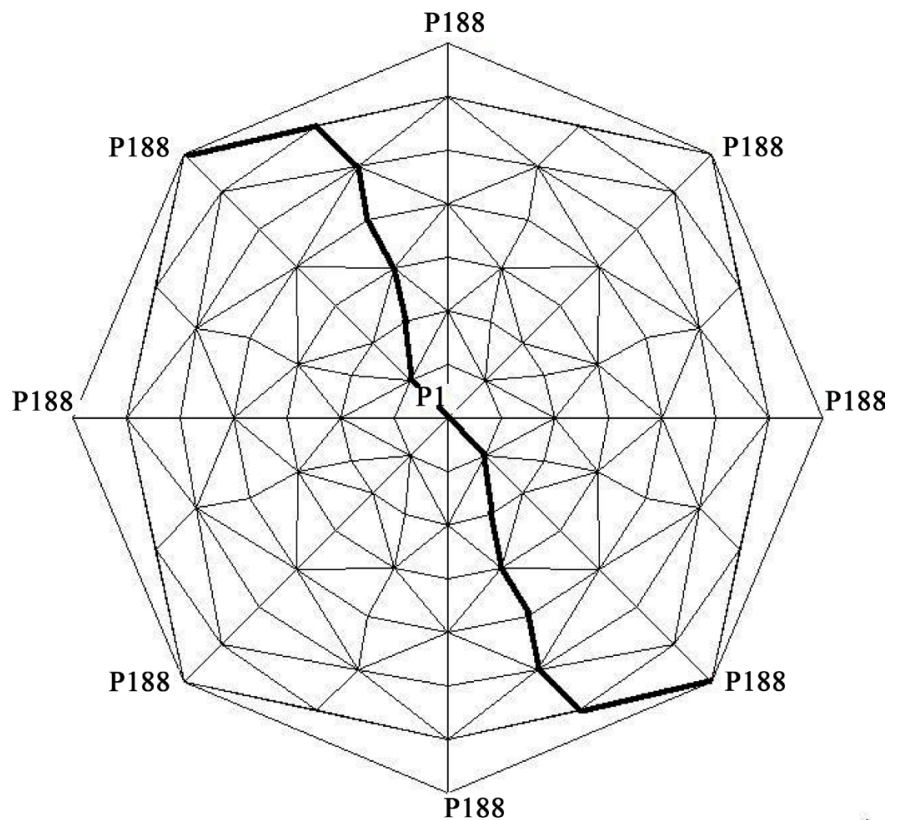

Figure 4. 2D-map of the spherical surface of the space object shown in Figure 2.

space points $\mathrm{P} 1$ and $\mathrm{P} 188$, among others.

We assume that of the $N_{e c}$ energy-carrying space points, $N_{\text {loop }}$ end up in geodesic loops (possibly at different surface layers). The remaining $N_{\text {radial }}$ points $\left(N_{\text {radial }}=N_{e c}-N_{\text {loop }}\right.$ ) cannot be prevented from periodically leaving the scope of the (narrow) quantum loop surface, resulting in an oscillating behaviour. These oscillating energy-carrying space points will leave the quantum loop only for a small distance before returning to the quantum loop. The set of energy-carrying space points belonging to the quantum loop have an overall vector of momentum with a specific direction (and size). The overall direction is preserved during and after the formation of the quantum loop. However, the $N_{\text {loop }}$ looping energy-carrying space points cannot contribute to the overall direction of momentum. The looping energy-carrying space points are direction-neutral. This leads to Proposition 15:

Proposition 13. The overall momentum (direction and amount) of the quantum loop and the momentum-related energy are determined by the sum of the momenta of the oscillating energy-carrying space points. The mass (energy) of the quantum object represented by the quantum loop is determined by the sum of the energies of the looping energy-carrying space points.

Notice that the total energy $E_{\text {total }}$ of the quantum loop is not simply the sum of the momentum energy $p \cdot c$ and the mass energy $m c^{2}$, but is determined by

$$
E_{\text {total }}=\sqrt{(p c)^{2}+\left(m c^{2}\right)^{2}} \text {. }
$$

\subsection{QFT within the Quantum Loop}

The second type of condition that must be satisfied for a quantum loop to form and remain stable concerns the QFT-related details of the quantum loop internal 
dynamics, and in particular the details of the interaction operator $\chi$ (see Section 6.3). The essential part of the quantum loop dynamics concerns the fermionic and bosonic energy-carrying space points. In each state update cycle of the process, the interaction operator $\chi$ is applied to the energy-carrying space points (see Specification 10). This means, the propagation of the energy-carrying space points is a continuous series of $\chi$ applications, i.e., a continuous series of Spread-out/Bundle processes. Instead of the simple example of a geodesic loop shown in Figure 4, a complex "geodesic loop network" develops.

The detailed $\chi$ function is derived from the rules of QFT, which describe the interaction (i.e., scattering) between particles. However, with the adaptation of the rules of standard QFT to the causal model of spacetime dynamics, a number of alternatives exist. Their impact on the final result of the collective behaviour process cannot be determined purely from mathematical calculations, and computer simulations are required to determine the optimal algorithm. The following list of questions will be answered with the help of computer simulations:

- Is there a minimal number of energy-carrying space points that is required to enable the collective behaviour process?

- Is there a minimum amount of energy of the collection of energy-carrying space points that is required to enable interactions and thus the collective behaviour process?

- What are the rules for the distribution of the energy (amplitude and frequency) to the multiple out-connections?

This is a major area for experimentation. The goal of maintaining compatibility with QFT establishes a frame within which alternative strategies are possible.

- If $\chi$ has only a single fermionic or bosonic in-connection, under what conditions is there only a single out-connection (i.e., an unchanged in-connection)?

- When more than one fermionic in-connection occur at a space point, is it acceptable to just let these pass the space point, or should a superposition of the in-connections be performed?

The major goals for the determination of the exact function of $\chi$ are 1) to enable the collective behaviour process; and 2) to ensure that the continuously occurring interactions between the energy carrying space points do not result in the dispersion of the overall energy of the quantum loop.

\subsection{Emergence of Elementary Particles}

The quantum loop dynamics described in Sections 7.1 and 7.2 results in the emergence of elementary particles (quantum loops that constitute composite quantum objects are briefly discussed in Section 8). The emergence of an elementary particle means 1 ) the emergence of a stable object that behaves like an elementary particle, and 2) the emergence of global particleattributes $\left(\Theta_{\text {mass }}, \Theta_{\text {charge }}, \Theta_{\text {spin }}\right)$ that are associated with the elementary particle, according to Definition 8.

Examples of the emergence of elementary particles in nature include the QFT 
scattering processes and the decay of composite quantum objects.

Emergence of mass The emergence of elementary particles includes the emergence of the mass of the particle (energy-carrying space points, the constituents of quantum loops, do not have a mass). In physics, the mass of an object is responsible for a number of effects. In a local causal model, it is not sufficient to specify a model for the emergence of an object-global attribute such as the mass. In addition, a causal model for the occurrence of the corresponding effects must be developed. In the causal model of quantum loops, two main effects associated with the mass of the emerged particle must be demonstrated: (i) the energy division of the emerged particle; and (ii) the gravitational field caused by the particle.

The total energy delivered by the collection of energy-carrying space points to the quantum loop is $E_{q l}$. Under the assumption that the emergence of the elementary particle does not result in a loss of energy, $E_{q l}$ is also the total energy of the emerged particle, $E_{\text {particle }}=E_{q l}$. The total energy $E_{\text {particle }}$ is composed of the mass energy $E_{m}=m c^{2} \quad(m=$ mass $)$ and the kinetic energy $E_{k}=p c \quad(p=$ momentum). According to relativity theory, we have

$$
E_{\text {total }}=\sqrt{E_{k}^{2}+E_{m}^{2}}=\sqrt{(p c)^{2}+\left(m c^{2}\right)^{2}}
$$

Thus, the emergence of the elementary particle must achieve a division of the total energy into $E_{k}$ and $E_{m}$, satisfying Equation (7). Proposition 13 associates the momentum-related energy $E_{k}$ with the oscillating energy-carrying space points, and the mass energy with the looping energy-carrying space points. This may achieve a subdivision of energy that satisfies Equation (7). In addition, however, the process of the emergence of mass has to satisfy a further condition, namely that the (rest-) masses of particles have a fixed value that depends only on the type of particle. In relation to Proposition 13, this means that the sum of the energies of the looping energy-carrying space points would require a critical value to obtain a stable quantum loop, that is, a stable elementary particle.

\subsection{Quantum Loop Evaporation}

The key characteristic of the quantum loop is the confinement of the energy-carrying space points within a small volume of curved space. The loop behaviour described above applies to fermionic and bosonic field types. In addition to these field types, in Section 6.1 the gravitational field $\psi_{G}$ and Schrödinger field $\psi_{S}$ are introduced. Fields of type $\psi_{G}$ and $\psi_{S}$ are continuously generated with the interaction operator $\chi(s p)$. In contrast to the fermionic and bosonic fields, the gravitational and the Schrödinger fields 1) do not dissipate energy from their source and 2) propagate without a preferred direction. As a result, the gravitational and Schrödinger fields are not confined within the quantum loop.

Proposition 14. The gravitational field $\psi_{G}$ and the Schrödinger field $\psi_{S}$ evaporate continuously from the quantum loop.

Evaporation of the gravitational field In the causal model of spacetime dy- 
namics described in [7] and [4] and in Section 4.3, quantum objects are treated as the sources of spacetime dynamics, that is, sources of the gravitational field. The assumption of quantum loops that constitute quantum objects is a refinement of the model described in [7] and [4]. The dynamics within the quantum loop consists of the continuous invocation of the interaction operator $\chi(s p)$ when the energy-carrying space points propagate from one space point to another. The interaction operator $\chi(s p)$ transforms the fields at the in-connections to those at the out-connections. The gravitational field is generated continuously, that is, with each application of the $\chi(s p)$ operator. The gravitational field generated within the quantum loop is not confined within the quantum loop, but evaporates from it.

Evaporation of the Schrödinger field The Schrödinger field $\psi_{S}$ takes (part of) the role of the wave function of quantum mechanics that follows the Schrödinger equation (see Equation (8)). Like the gravitational field, the Schrödinger field arises as a by-product of each application of the $\chi(s p)$ operator, that is, with the propagation of the energy-carrying space points. Further details of the Schrödinger field are given in Section 8.

\subsection{Quantum Loops of Composite Quantum Objects}

The focus of this article is on quantum loops that constitute elementary particles; quantum loops that constitute composite quantum objects are not discussed in detail. Nevertheless, the author believes that the following characteristics of quantum loops are also applicable to composite quantum objects:

- internal dynamics that constitutes a loop processes

- collective behaviour of the elements (e.g., particles) that make up the composite quantum object

- phase transition to (semi-) stable states

In general, composite quantum objects can develop towards multiple (semi-) stable states depending on the overall energy of the quantum object. These (semi-) stable states are called eigenstates.

\subsection{Movement of Quantum Objects}

When quantum objects emerge in the form of quantum loops, part of the available energy ends up in the mass of the quantum object, while the remainder determines the momentum of the quantum object, that is, the movement in space.

In addition to the momentum of the quantum object, further external influences and parameters determine the actual trajectory of the quantum objec (see below).

\section{The Dynamics of Quantum Objects-Quantum Mechanics}

Overall objective of the present section is the description of a local causal model of QT that is based on the causal model of spacetime dynamics described in Section 4 and on the model of quantum field dynamics described in Sections 5, 6 
and 7. The description focuses on the dynamics of quantum objects, that is, the propagation of quantum objects in their environment and the interaction between quantum objects. The internal dynamics within composite quantum objects is not discussed in the present article. ${ }^{8}$

\subsection{Waves and/or Particles?}

In the Copenhagen interpretation of standard quantum mechanics, the particle and wave models are complementary, making the overall model complex and obscure. In the causal model of QT/QFT, the major components of the system state are: 1) space; 2) fields; and 3) quantum objects. Quantum objects are specified in Definition 8 as a collection of particles, including elementary particles. The dynamics of quantum objects takes place in fields, which are represented by waves. A clear separation between quantum objects (i.e., collections of particles) and their environment, in the form of fields and waves, is comparable to the assumptions used in the de Broglie-Bohm theory (see [12] and [13]), in which the particle configuration is separated from the wave function. This is in contrast to standard QT, where the Hamiltonian in the Schrödinger equation combines the particle configuration(s) with their environment. The separation of particles from their environment is disturbed by the assumption that quantum loops (representing quantum objects) continuously modify their environment by issuing Schrödinger fields $\psi_{S}$ and gravitational fields $\psi_{G}$.

\subsection{The Role of the Schrödinger Equation}

The model of the quantum loop described in Section 7 assumes that the quantum object (established by a quantum loop) continuously evaporates the Schrödinger field $\psi_{S}$ in addition to the gravitational field. The Schrödinger field that is generated this way interacts with the moving quantum object and thereby is a major contributor to the determination of the trajectory of the quantum object in space. This model of the wave function is close to the de-Broglie-Bohm theory (see [12] and [13]). Appendix B gives a comparison of the causal model of QT with the de Broglie-Bohm theory.

In standard quantum theory, the Schrödinger equation

$$
i \frac{\mathrm{d}}{\mathrm{d} t}|\Psi(t)\rangle=\hat{H} \mid \Psi(t)
$$

determines the wave function, i.e., the propagation of the quantum object in space. For a typical Hamiltonian, $\mathrm{H}$, the time-dependent one-dimensional Schrödinger equation becomes

$$
i \hbar\left(\frac{\partial \Psi}{\partial t}\right)=\frac{-\hbar^{2}}{2 m} \frac{\partial^{2} \Psi}{\partial x^{2}}+V \Psi .
$$

As can be seen from Equation (8), the parameters used in the Schrödinger equation represent the complete (sub-)system considered, the collection of par-

${ }^{8}$ The internal dynamics within elementary particles is discussed in Section 7, Quantum Loops. 
ticles and the environment, which is represented by $V$. Another point of interest for a causal model can be seen by looking at the "time-independent" version of the Schrödinger equation

$$
(E-V) \Psi=\frac{-\hbar^{2}}{2 m} \frac{\partial^{2} \Psi}{\partial x^{2}} .
$$

Equation (9) expresses the conservation of total energy $E$ as the sum of the potential energy $V$ and the kinetic energy, represented by the right-hand side of Equation (9). This equation implies that the kinetic energy expressed by the wavelength of $\Psi$ increases with the potential $V$, in accordance with Proposition 11.

In the causal model of QT/QFT, in the same way as in the de Broglie-Bohm theory, the equation of motion of the particle, the Schrödinger field $\psi_{S}$ and other environmental influences (see Section 8.3) determine the deterministic propagation of the particles.

\subsection{Interactions with the Environment}

In contrast to earlier interpretations of QT, environmental influences must be taken into account more seriously in modern QT. In [14] Schlosshauer writes: "... these experiments have shown that any observed disappearance of quantum coherence and interference can be attributed to the environment, that is, to decoherence.". In addition to the momentum of the quantum object, additional external influences and parameters determine the actual trajectory of the quantum object, including:

- Interaction with the gravitational field, i.e., the space curvature

- Interaction with the Schrödinger field $\psi_{S}$

- Weak interactions with other quantum objects (decoherence)

- Strong (destructive) interactions with other quantum objects.

These (destructive) interactions take place between quantum objects, and typically between the elementary particles described by the rules of QFT (e.g. the scattering matrix, particle creation/annihilation). This implies that the interacting particles are de facto destroyed (i.e., annihilated). Although they may be resurrected (i.e., created again), this would imply a new wave function or at least a nonlinear change in the interacting quantum objects (i.e., a collapse of the wave functions). In the following, these types of interactions are referred to as $Q F T$ interactions.

\subsection{The QT Measurement Problem}

Theories and models of QT measurement are typically called interpretations of QT, which appear to be less important add-ons to QT. In contrast, the author believes that a model of the QT measurement is essential for a deeper understanding of QT. This view is supported by Maudlin's famous formulation of the QT measurement problem, in which he listed three basic assumptions of standard QT and showed that these are partly contradictory. In [15], Maudlin for- 
mulated the QT measurement problem in form of a trilemma, in which he claimed that the following three statements are mutually incompatible:

1) The wave function is a complete description of the state of a QT system.

2) The wave function always evolves in accordance with a linear dynamical equation (e.g., the Schrödinger equation).

3) Each measurement has a definite result which is one of the possible results for which the probability distribution satisfies the Born rule.

Maudlin also gives variations of these contradictory claims that are also contradictory. Thus, we need to give up at least one of these three statements in order to come up with a non-contradictory theory of QT measurement (and hence an acceptable interpretation of QT). In fact, the major interpretations of QT can be classified based on which of the three statements is discarded. In the causal model of QT/QFT, the claims that (i) the wave function is a complete description of the state of the system and (ii) the wave function always evolves in accord with the Schrödinger equation are not supported. As described in Sections 4-7, in the causal model, the system state is more extensive than the wave function. ${ }^{9}$

In addition, the causal model described here originated from the authors experience that linear equations such as the Schrödinger equation are not sufficient to express complex causal relationships of the type occurring in QT/QFT. A determination of which of the basic QT claims are not supported by the causal model does not of course imply the specification of a causal model/interpretation of QT measurement; in a causal model of QT/QFT, measurements must be part of the normal causal development of the system that is considered. In a causal model that adheres to the specification given in Section 2, this means three requirements: i) the laws of physics of the causal model must not contain any specific laws that refer to measurement situations (e.g., "IF measurement THEN... "); ii) the laws of physics must not refer to any parameters that are not contained in the system state; and iii) the system state must not contain any parameters or components that are not subject to the causal development. These requirements apply to causal models in general and, for example, exclude a reference to a thing called an "observer" in the causal model. In local causal models, further requirements are obvious and are therefore not further addressed. Interactions between the measured quantum object and the measurement apparatus play a key role in a model of the QT measurement process, which is an integral part of the normal causal model of QT.

Proposition 15. A QT measurement requires at least one QFT-interaction between the measured quantum object and the measurement apparatus. (The term "QFT interaction" has been introduced in Section 8.3 above.)

For an explanation of claim (iii) above, i.e., the selection of a definite measurement result, a further proposition can be established:

Proposition 16. Measurement in $Q T$ is, in general, the measurement of the

${ }^{9}$ It may be possible to specify a subset of the system state of the causal model and to explain the major QT features in terms of this subset. However, it is claimed that such a subset would not be sufficient to explain QT measurement. 
position of the measured quantum object. Other attributes that are indirectly measured are typically deduced from the measured position. If the measurement of the position of the quantum object has the goal of determining the value of another observable, the objective of the measurement arrangement is to enable a unique mapping of the position to the desired observable. Having determined a definite measurement result, the question remains as to why the other alternative measurement results are also eliminated from occurring (possibly later). Three main types of models have been proposed, based on various interpretations of QT: (i) the collapse of the wave function; (ii) the many worlds theory (see [16]); and (iii) the de Broglie-Bohm theory.

As described above, in a similar way to the de Broglie-Bohm theory, the causal model of QT/QFT separates (i) the actual configuration of the quantum object from (ii) the wave function (i.e., the Schrödinger field $\psi_{S}$ in the causal model of QT/QFT). The measurement applies to the actual configuration. At the moment of measurement (i.e., when the QFT interaction occurs) there are no alternative eigenstates to be eliminated.

\subsection{The Role of the Probability Amplitude}

In [17], Feynman describes the fundamental principle of quantum mechanics $(\mathrm{QM})$ as follows: "It has been found that all processes so far observed can be understood in terms of the following prescription: To every process there corresponds an amplitude; with proper normalisation the probability of the process is equal to the absolute square of this amplitude".

The square of the probability amplitude $|\psi|^{2}$ gives the probability of measurement of a particular value of a QT observable. According to Proposition 16, $|\psi(x)|^{2}$ first gives the probability of finding the particle at position $\mathrm{x}$. If an observable other than the position is to be measured,, it is the task of the measurement arrangement to enable a unique mapping of the position to the desired observable. In standard QT, the statement "the probability amplitude $|\psi|^{2}$ gives the probability of the measurement of a particular value of a QT observable" is called the Born rule. In the de Broglie-Bohm theory and the causal model of QT/QFT, this statement requires a causal formulation; this is called the quantum equilibrium hypothesis (see [11]), and is as follows:

Proposition 17. In quantum equilibrium the system is in a state such that the position distribution $\rho$ of a particle described by the wave function $\psi(x, t)$ is $\rho=|\psi(x, t)|^{2}$. Thus, the Born rule is not considered a basic law of QT; instead, the condition $\rho=|\psi(x, t)|^{2}$ is the result of a causal process with possible phases in which the condition is not satisfied. In the causal model of QT/QFT, processes that are assumed to lead towards a quantum equilibrium are related to the model of quantum loops described in Section 7. This includes

- the emergence of elementary particles (Section 7)

- the emergence of composite quantum objects (briefly described in Section 7.5)

- the propagation of quantum objects 
- the transition to eigenstates

\subsection{Entanglement}

The biggest obstacle to the construction of a local causal model of QT/QFT is the local causal model of QT entanglement. QT entanglement is understood here as entanglement represented by the EPR experiment (see [18]). The realisation of the EPR experiment by Aspect (see [19]) resulted in confirmation of the QT predictions, which also implied a violation of Bell's inequality (see [20]). Due to the violation of Bell's inequality in the EPR experiment, QT physicists (including Bell) concluded that a local causal model of QT is not feasible. ${ }^{10}$ In [5] and [8], a local causal model of QT/QFT is presented in which instead of space-point locality, object-locality is solely used. Object-locality means that actions of the causal model may refer to object global components. In the causal model of the EPR experiment, this means that the two entangled particles of the EPR experiment are considered to constitute a common quantum object with a common (object-global) wave function. However, it is not clear to the author whether this model of an "entanglement quantum object" is compatible with the model of quantum loops representing quantum objects, as described in Section 7. It is possible that such an entanglement within quantum objects would, at least, imply a size limit for quantum objects based on quantum loops.

\subsection{Decoherence}

In Section 8.3, four types of interactions between a quantum object and the environment are described. Decoherence (i.e., "weak interaction" with other quantum objects) is one of these possible types of interaction. In decoherence theory (see [14]), this means decoherence of the quantum objects wave function. The wave function of the quantum object-1 $\psi_{1}$ is coupled with the wave function of the quantum object-2 $\psi_{2}$ to become (part of) a common wave function,

$$
\psi_{\text {common }} \leftarrow \psi_{1} \otimes \psi_{2} \text {. }
$$

In the causal model of QT/QFT, the model of decoherence is largely the same as in standard QT, except for the following differences:

- decoherence is just one of the possible types of interactions; in particular, QFT interactions are also important in the model of the QT measurement process,

- in the causal model of QT/QFT, decoherence is not only the interaction between two quantum objects, but it is the interaction between the Schrödinger field $\psi_{S}$ generated by particle-1 and the Schrödinger field of the environment. ${ }^{11}$

\footnotetext{
${ }^{10}$ This motivated the author to develop a formal definition of a (local) causal model and to develop a (local) causal model of QT/QFT.

${ }^{11}$ This is not just a different terminology: it reflects a generalisation that is applicable to further types of particle interactions with the environment.
} 
- The operator $\otimes$ in “ $\psi_{\text {common }} \leftarrow \psi_{1} \otimes \psi_{2}$ " is a simplification that needs to be refined with a causal model.

\section{Discussion}

\subsection{Quantum Gravity}

Loop quantum gravity and its derivatives, spin networks and causal dynamical triangulation are approaches that influenced the work described in this article. This article describes a causal model of both spacetime dynamics and QT/QFT. For these reasons, this model can be considered to be another candidate theory towards quantum gravity. However, the author does not consider this a model of quantum gravity for two reasons: (i) it is not clear exactly what is expected from a model of quantum gravity; and (ii) the work described in this article lacks the maturity of existing theories on quantum gravity. Nevertheless, this model does address a number of questions that are typically addressed in theories of quantum gravity (see [21]):

1) Discretisation

The models of spacetime dynamics and of QT/QFT contain discrete units of space, time and the derived discretisation (quantisation) of paths and waves.

2) Approaches to quantising gravitation

No attempt has been made to quantise gravitation. Quantum behaviour is restricted to quantum objects, which emerge from (non-quantised) space and energy-carrying space points.

3) Gravitation between particles

According to the model of spacetime dynamics, significant curvature of space (i.e., gravitation) already exists around particles.

4) No quantum behaviour of the gravitational field, i.e., no uncertainty and no probabilistic measurement results

This is explicitly endorsed in the causal model.

5) Singularities of GRT

The singularities at the minimal sources and at the centres of gravitation are avoided in the discretised causal model. The time dilation singularity at the horizon of the black hole is not supported by the causal model.

6) Information loss from black holes

The concept of "(no) information loss" is not supported by the causal model.

7) Perturbative quantum gravity is not renormalisable

Pertubative QFT, pertubative quantum gravity and renormalisation are (assumed to be) not required in the discretised causal model.

8) Gravitons

In the model of the emergence of elementary particles (quantum loops), the emergence of gravitons out of the gravitational field is not anticipated.

\subsection{Relation to Loop Quantum Gravity}

Although the causal model of spacetime dynamics and QT/QFT is not seen by 
the author as being on an equal footing with loop quantum gravity (see [22] [23]), it is nevertheless worth pointing out the major differences between the two in terms of the approach and the concepts used:

- The role of quantisation

In the causal model, spacetime is not quantised (in the sense understood in QT/QFT), and instead is simply discretised. Quantisation is applied only to quantum fields and quantum objects.

- The interpretation of QT

Since loop quantum gravity applies quantisation at the level of the elementary structure of spacetime, the type of QT interpretation affects the spacetime model of loop quantum gravity. According to [23], loop quantum gravity assumes a relational interpretation of QT. The causal model of spacetime dynamics and QT/QFT implies an interpretation of QT that may be viewed as a "collapse theory". However, in the causal model, this collapse does not apply to the wave function (i.e., the Schrödinger field), but to the quantum object.

- The elementary structure of space and time

As mentioned in [4], the model of spacetime dynamics described in Section 4 and in [4] is derived from causal dynamical triangulation (CDT, see [24]). Unlike in CDT, there is more flexibility in the causal model of spacetime dynamics in terms of the elementary units of space (structure, volume). In addition, the space-time relationship is defined in a way that eliminates time dilations at the elementary level.

A (slightly) more detailed comparison between loop quantum gravity and the causal model of spacetime dynamics and QT/QFT is given in Appendix D.

\subsection{The Higgs Field and Higgs Particle}

In Section 6.1, where the different field types are discussed, the Higgs field is not mentioned, the author has not yet determined what the role of the Higgs field should be within the causal model of quantum field dynamics. From the perspective of standard QFT, the Higgs field would be considered as a QFT field (with certain special properties such as spin zero). Within the model of quantum field dynamics (Section 6), the Higgs field could also be considered a secondary field, that is, a field that does not carry energy, like the gravitational field or the Schrödinger field, does not carry energy. Treating the Higgs field as a secondary field type would give a simple explanation for the general assumption that the Higgs field fills the whole universe. It would also enable an apparently simpler explanation of the assumption that the Higgs field gives rise to the masses of particles. However, the author believes that the Higgs field should not be considered a secondary field, unless its relation to the other secondary field types (gravitational and Schrödinger field) can be defined. One (radical) model would be that the three field types (gravitational, Schrödinger and Higgs) are all the same.

After the role of the Higgs field has been clarified, it should be discussed whether this implies the existence of a Higgs particle. 


\subsection{Cosmology}

The causal models presented in this article explicitly focus on the very low end of the scale. Nevertheless, some possible implications can be seen for the area cosmology (i.e., at the very high end of the scale). Two main items of the model of spacetime dynamics may enable new interpretations and new models in cosmology:

- The gravitational length dilation, as described in Section 4.2.

- The accumulation of space (curvature) changes resulting from multiple sources, as described in Section 4.3.

Both of these items enable new interpretations of astronomical observations. One example of a possible alternative interpretation of an astronomical observation is the observation of the "flat galactic rotational curves" (see, for example [25] and [26]). The explanation that is favoured by most astrophysicists is the existence of "dark matter". In [7] a proposal is described in which flat galactic rotational curves are explained by the gravitational length dilaation.

\subsection{Relation to String Theory}

No attempt has been made to adopt any of the concepts of string theory. The only common ground with string theory observed by the author is the possible string interpretation of the space point connections.

\section{Conclusions}

This article covers a wide range of subjects, using a bottom-up approach. Beginning with a model of spacetime dynamics, a model of quantum field dynamics and finally a model of quantum theory are presented. The work described in the article was influenced by many existing theories, both established and controversial. In the area of spacetime dynamics, the primary influence came from loop quantum gravity and its derivatives, i.e., spin networks and causal dynamical triangulation. In the proposed model of quantum mechanics, similarities to the de Broglie-Bohm theory arose. In each area, however, the model contains significant deviations from the source theories. Although the areas that have been addressed span a wide range, there are two subjects that are fundamental to the whole article: (i) causal models and (ii) quantum loops. Over the past decade, the author has focused on causal models, and is increasingly convinced that (only) causal models enforce complete models and lead to solutions that are otherwise easily overlooked.

The overarching topic of the present paper is quantum loops, which were found to offer a possible model for nonlinear processes at the very lowest scale, resulting in collective behaviour of the elementary elements of space and quantum fields. Due to the size constraints on the paper, there are many areas that have been only briefly addressed, and there are also areas that require further work to verify and refine the proposed model (see Appendix A), including several aspects that can be refined and verified only by use of computer simulations. 
Models that contain discrete units of space and time, nonlinear processes and collective behaviour processes cannot be evaluated using differential equations alone, and this includes the topics of quantum loops and quantum equilibrium. Computer simulations are planned on the subject of quantum loops.

\section{Conflicts of Interest}

The author declares no conflicts of interest regarding the publication of this paper.

\section{References}

[1] Diel, H.H. (2013) Problems and Possible Solutions with the Development of a Computer Model of Quantum Theory. World Academy of Science, Engineering and Technology, 7, 221-225.

[2] Diel, H.H. (2013) On the Computability of Quantum Theory. International Journal of Computational Physical Sciences, 4, 7-22.

[3] Diel, H. (2016) The Completeness, Computability, and Extensibility of Quantum Theory. http://arXiv:1512.08720

[4] Diel, H.H. (2018) A Local Causal Model of Spacetime Dynamics. Open Access Library Journal, 5, e4957.

[5] Diel, H.H. (2017) Collective Behavior in a Local Causal Model of Quantum Theory. Open Access Library Journal, 4, e3898.

[6] Schutz, B.F. (2009) A First Course in General Relativity. Cambridge University Press, New York. https://doi.org/10.1017/CBO9780511984181

[7] Diel, H.H. (2018) Emerging Quantum Fields Embedded in the Emergence of Spacetime. https://doi.org/10.20944/preprints201804.0379.v1

[8] Diel, H. (2016) Quantum Objects as Elementary Units of Causality and Locality. http://arXiv:1609.04242v1

[9] Wong, C.W. (1991) Introduction to Mathematical Physics, Methods \& Concepts. Oxford University Press, Oxford.

[10] Strassler, M. (2013) Conversations about Science with Theoretical Physicist M. Strassler.

http://profmattstrassler.com/articles-and-posts/particle-physics-basics/Higgs/6.Fiel dsOfParticular Significance.html

[11] Dürr, D., Goldstein, S. and Zanghi, N. (1992) Quantum Equilibrium and the Origin of Absolute Uncertainty. Journal of Statistical Physics, 67, 843-907. https://doi.org/10.1007/BF01049004 https://arxiv.org/abs/quant-ph/0308039

[12] Dürr, D., Goldstein, S., Tumulka, R. and Zanghi, N. (2003) Bohmian Mechanics and Quantum Field Theory. Physical Review Letters, 93, Article ID: 090402.

[13] Dürr, D., Goldstein, S., Tumulka, R. and Zanghi, N. (2005) Bell-Type Quantum Field Theories. Journal of Physics A, 38, R1.

[14] Schlosshauer, M. (2007) Decoherence and the Quantum-To-Classical Transition. Springer Verlag, Berlin.

[15] Maudlin, T. (1995) Three Measurement Problems. Topoi, 14, 7-15. https://doi.org/10.1007/BF00763473

[16] Everett, H. (1957) “Relative State” Formulation of Quantum Mechanics. Reviews of 
Modern Physics, 29, 454. https://doi.org/10.1103/RevModPhys.29.454

[17] Feynman, R.P. (1961) The Theory of Fundamental Processes. Westview Press, Boulder, CO.

[18] Bell, J.S. (1964) On the Einstein-Podolsky Rosen Paradox. In: Speakable and Unspeakable in Quantum Mechanics, Cambridge University Press, Cambridge.

[19] Aspect, A., Dalibard, J. and Roger, G. (1982) Experimental Test of Bell's Inequalities Using Time-Varying Analyzers. Physical Review Letters, 49, 1804-1807. https://doi.org/10.1103/PhysRevLett.49.1804

[20] Bell, J.S. (1984) Beables for Quantum Field Theory. In: Speakable and Unspeakable in Quantum Mechanics, Cambridge University Press, Cambridge.

[21] Hossenfelder, S. (2016) Quantengravuitation auf dem Weg zur Wissenschaft, Spektrum der Wissenschaft.

[22] Gambini, R. and Pullin J. (2011) A First Course in Loop Quantum Gravity. Oxford University Press, Oxford.

[23] Rovelli, C. (2017) Reality Is Not What It Seems: The Journey to Quantum Gravity. Penguin, London.

[24] Loll, R., Ambjorn, J. and Jurkiewicz, J. (2005) The Universe from Scratch. http://arXiv:hep-th/0509010

[25] Turyshev, S. and Toth, V. (2010) The Pioneer Anomaly. Living Reviews in Relativity, 13, 4. https://doi.org/10.12942/lrr-2010-4

http://www.livingreviews.org/lrr-2010-4

[26] Wikipedia (2019) Galaxy Rotation Curve.

[27] Dabin, R. (2009) De Broglie-Bohm Theory: A Hidden Variables Approach to Quantum Mechanics. 


\section{Appendix}

\section{A1. Major Aspects That Remain to Be Addressed/Solved}

1) Spacetime dynamics: The accumulation of space changes caused by multiple sources

2) Spacetime dynamics: The variable expansion factor with the emergence of space

3). Spacetime dynamics: The singularity of time dilation at the Schwarzschild radius

4) Quantum field dynamics: The relation between field types (bosonic, fermionic, gravitational, Schrödinger, Higgs)

5) Quantum field dynamics: The role of spin in the causal model

6) Quantum Loops: The detailed conditions for the emergence of quantum loops

7) Quantum Loops: A model of the emergence of particle generations (including mass values)

8) Quantum Loops: The emergence of composite quantum objects

9) Quantum Loops: The details of the generation of the Schrödinger field

10) Quantum Mechanics: The local causal model of EPR experiment

11) Quantum Mechanics: The uncertainty principle

\section{A2. Comparison of the Causal Model of QT/QFT with the De Broglie-Bohm Theory}

Although the causal model of QT described in Section 8 is influenced by the de Broglie-Bohm theory, there are also significant differences.

The two basic constituents of the de Broglie-Bohm theory are:

1) The Schrödinger equation that describes the wave function $\psi(x)$ and,

2) The guiding equation that describes the particle configuration

$$
Q_{i}-\frac{\mathrm{d} Q_{i}}{\mathrm{~d} t}=\frac{\nabla_{i} S}{m i}
$$

Another important ingredient is the "quantum equilibrium" hypothesis:

The position distribution $\rho$ of a system that is described by the wave function $\psi$ is $\rho=|\psi|^{2}$.

Table A1 shows a comparison of the causal model of QT/QFT with the de Broglie-Bohm theory.

Comments on Table A1:

- (1) The causal model of QT/QFT supports space point locality. The local causal model as requested by the EPR-experiment is supported only, if the entangled particles belong to the same (composite) quantum object. On the other hand, only a limited size of quantum objects is considered feasible.

- (2) The wave function and $\psi_{S}$ is a global object like the space. However, the local causal model of spacetime dynamics and of quantum field dynamics requires (and supports) local dynamics only. 
Table A1. Comparison of the causal model of QT/QFT with the de Broglie-Bohm theory.

\begin{tabular}{|c|c|c|}
\hline & de Broglie-Bohm theory & causal model of QT/QFT \\
\hline implies interpretation of QT & yes & yes \\
\hline causal model & yes & yes \\
\hline deterministic model & yes & yes \\
\hline Local causal model & no & $\begin{array}{l}\text { yes, but limited size } \\
\text { of quantum object }{ }^{\star} 1\end{array}$ \\
\hline $\begin{array}{l}\text { separation of particle configuration } \\
\text { and environment }\end{array}$ & quantum object/wave function & quantum object $/ \psi_{S}+\psi_{G}+\cdots$ \\
\hline $\begin{array}{l}\text { global wave function } \\
\text { for universe }\end{array}$ & yes & $\begin{array}{c}\text { global } \psi_{s} \text {, but local } \\
\text { dynamics }{ }^{*} 2\end{array}$ \\
\hline model of measurement & yes & yes (see Section 8.3) \\
\hline $\begin{array}{l}\text { measurement implies interaction } \\
\text { with measurement apparatus }\end{array}$ & yes & yes \\
\hline $\begin{array}{l}\text { measurement is typically position } \\
\text { based }\end{array}$ & yes & yes \\
\hline measurement: role of observer & no & no \\
\hline Collapse of wave function & $\begin{array}{l}\text { other eigenstates continue to } \\
\text { exist }{ }^{*} 7\end{array}$ & $\begin{array}{l}\text { collapse of the quantum } \\
\text { object }\end{array}$ \\
\hline observables & no & no \\
\hline non-local mass & yes & $\begin{array}{c}\text { mass is a quantum } \\
\text { object-global attribute }{ }^{*} 3\end{array}$ \\
\hline $\begin{array}{l}\text { particles act back on } \\
\text { wave function }\end{array}$ & no & yes $^{*} 4$ \\
\hline Entanglement & non-local & quantum object local $* 5$ \\
\hline Quantum equilibrium hypothesis & yes & $\begin{array}{c}\text { emergence of stable } \\
\text { collective behaviour }{ }^{*} 6\end{array}$ \\
\hline absolut space and time & yes & yes \\
\hline $\begin{array}{l}\text { special interpretation of } \\
\text { uncertainty principle }\end{array}$ & yes & $?$ \\
\hline complementarity & no & no \\
\hline spin treatment & $?$ & $?$ \\
\hline contextualisation & yes & $?$ \\
\hline Support of many worlds & no $* 8$ & no \\
\hline
\end{tabular}

- (3) The mass of an elementary quantum object emerges together with the quantum object.

- (4) The Schrödinger field $\psi_{S}$ (like the gravitational field $\psi_{G}$ ) evaporates from the quantum object.

- (5) The causal model of QT/QFT supports EPR-like entanglement only if the entangled particles belong to the same (composite) quantum object.

- (6) The emergence of quantum equilibrium is comparable to the emergence of a stable collective behaviour, as, for example, with the quantum loop. 
- (7) In [27] "These other eigenstates exist and still have ontological status but have no influence on the actual configuration $(x(t), y(t))$ ". How are these other eigenstates prevented from being measured?

- (8) Some physicists (e.g., H.D. Zeh and D. Deutsch) say that the de Broglie-Bohm theory is a many worlds theory. The author doubts that this is a correct interpretation of the de Broglie-Bohm theory and/or of the many worlds theory.

\section{A3. Time, Length and Energy Dilation with the GPS Satellite}

Table A2 shows the time, length and energy dilation for an object within a gravitational field with a single source (i.e., the Schwarzschild metric). The object (e.g. a GPS satellite) is assumed to be in an orbit at a sufficient distance from the source of the gravitational field.

Comments on Table A2:

- The column "Standard GRT" contains what the author understands as the GRT interpretation with most physicists. Apparently, some items are still controversial.

- The numbers given in the examples are not claimed to be realistic.

- With the "frequency of light" and "wavelength of light" for "standard GRT" (and a reduced speed of light) it is not clear to the author whether the energy reduction results in (a) a reduced frequency, (b) an increased wave length, or (c) both.

\section{A4. Comparison with Loop Quantum Gravity}

Comments on Table A3:

- (1) The causal model of spacetime dynamics and QT/QFT implies an interpretation of QT that may be viewed as a "collapse theory". However, in the

Table A2. Time, length and energy dilations.

\begin{tabular}{|c|c|c|c|c|c|}
\hline & $\begin{array}{l}\text { Pre-GRT } \\
\text { Example }\end{array}$ & $\begin{array}{c}\text { Standard GRT: } \\
\text { dilated time, } \\
\text { variable c }\end{array}$ & $\begin{array}{l}\text { Example } \\
F_{1}=0.98\end{array}$ & $\begin{array}{l}\text { Causal model: } \\
\text { dilated length, } \\
\text { time }\end{array}$ & $\begin{array}{l}\text { Example } \\
F_{1}=0.98\end{array}$ \\
\hline satellite turnaround time, $t s$ & 100,000 & $t s_{d}=t s / F_{1}$ & 102,000 & $t s_{d}=t s / F_{1}$ & 102,000 \\
\hline orbit length, $l$ & $1,000,000$ & $l_{d}=l$ & $1,000,000$ & $l_{d}=l / F_{1}$ & $1,020,000$ \\
\hline satellite speed, $v s=1 / t s$ & 10 & $v s \cdot F_{1}$ & 9.8 & $v s$ & 10 \\
\hline speed of light, $c$ & 300 & $c \cdot F_{1}$ & 294 & $c$ & 300 \\
\hline $\begin{array}{l}\text { turnaround time } \\
\text { of light, } t l=1 / c\end{array}$ & 3333 & $t l_{d}=t l / F_{1}$ & 3400 & $t l / F_{1}$ & 3400 \\
\hline $\begin{array}{c}\text { Kinetic energy of } \\
\text { satellite, } \quad k=1 / 2 m v^{2}\end{array}$ & $\begin{array}{c}50 \\
(m=1)\end{array}$ & $k \cdot F_{1}^{2}$ & 48.02 & $k$ & 50 \\
\hline Frequency of light $v$ & 150 & $v_{d}=v \cdot F_{1}$ & 147 & $v_{d}=v \cdot F_{1}$ & 147 \\
\hline Wave length of light $w l=c / v$ & 2 & $\left(c \cdot F_{1}\right) /\left(v \cdot F_{1}\right)=c / v$ & 2 & $c /\left(v \cdot F_{1}\right)$ & 2.04 \\
\hline
\end{tabular}


Table A3. Comparison of the causal model of QT/QFT with loop quantum gravity.

\begin{tabular}{ccc}
\hline & Loop Quantum Gravity & Causal model of QT/QFT \\
\hline $\begin{array}{c}\text { QT behaviour with } \\
\text { basic spacetime } \\
\text { space/time } \\
\text { Loops at }\end{array}$ & yes & no \\
disintegrated & disintegrated \\
snterpretation of QT & relational, & quantum field level \\
non-deterministic & causal, (non-) deterministic * 1 \\
Time & discrete units of volume, area, line & discrete space points, connections \\
levels of time & not an “object" & not a component of system state *2 \\
time-less gaps & (1) sequence of events & (1) constant suti * 3 \\
space(-time) dynamics & probabilistic, non-deterministic & local causal, deterministic \\
\hline
\end{tabular}

causal model, this collapse does not apply to the wave function (i.e., the Schrödinger field), but to the quantum object. In addition, commonalities with the de Broglie-Bohm theory exist.

- (2) As described in Section 2, in the causal model (in general) time is not a component of the system state, but an inherent feature of the physics engine.

- (3) The causal model assumes a uniform time progression in constant state update time intervals (suti) at the elementary level. Differing clock speed and proper time (as defined with GRT) applies to processes that execute in space. 